\title{
Salud mental en trabajadores expuestos COVID-19
}

\author{
Mental health in workers exposed to COVID-19 \\ Johana Acosta-Quiroz ${ }^{1, a}$, Sebastián Iglesias-Osores ${ }^{1, b}$
}

\section{Señores Editores:}

Los casos de COVID-19 van en aumento; enfermeras, médicos, biólogos y trabajadores de salud en general se ven expuestos a un mayor riesgo de contraer esta enfermedad (1). La gran carga de trabajo, los escases de equipos de protección personal, la falta de medicamentos específicos puede aumentar la carga mental (2). Los trabajadores de salud que constantemente participan en el diagnóstico y tratamiento de pacientes con COVID-19 están expuestos a desarrollar síntomas de ansiedad y estrés (3). La fácil transmisión del virus y las tasas de muerte asociadas a este pueden aumentar la percepción de peligro y estrés en el personal de salud (4). El objetivo de esta carta es dar a conocer los problemas que conllevan el estrés en el personal de salud.

La grave situación causo en los trabajadores sanitarios de Wuhan síntomas depresivos, estrés, ansiedad, insomnio, enojo y miedo, estos problemas de salud mental afectan la capacidad de toma de decisiones y pueden tener un efecto negativo en su vida (5). Durante el brote de SARS en 2003 estudios reportaron que los trabajadores de salud sentían temor de contagiar a sus familiares y amigos (6). Además de síntomas de ansiedad y depresión que podrían tener repercusiones en su salud mental, incluso un año después del brote del SARS los trabajadores de salud tenían un nivel elevado de estrés y angustia (2). Tener un trabajo de alto riesgo, el estar en contacto con personas infectadas y estar aislado son causas comunes de trauma (7). Cuidar la salud mental de los trabajadores sanitarios sería de gran importancia para controlar la epidemia (5). Los ataques de pánico también son una respuesta al estrés debido al trabajo excesivo a causa del brote de coronavirus (2).

Se tienen que tomar acciones para proteger la salud mental de los trabajadores sanitarios, un claro ejemplo es China que redujo la presión sobre médicos y enfermeras enviando más personal y ofreció orientación práctica estableciendo equipos de intervención psicológica para pacientes y trabajadores de salud (8). Esta pandemia probablemente traerá como consecuencia altas tasas de estrés postraumático, depresión y otras enfermedades de salud mental en los trabajadores de salud y el personal esencial, por estas razones debe existir un monitoreo e identificación temprana de las personas en riesgo (9). Además debería de haber una mayor inversión en herramientas de salud mental para atender y proteger al personal que son los que se encuentran en la primera línea de acción de respuesta la enfermedad (10). El gobierno peruano ha puesto esfuerzo en dar apoyo al personal por líneas de atención telefónica y grupos de voluntarios hacen lo mismo. La presión del personal de salud es tal que también se ven hostigados por los mismos pobladores temiendo contagio por parte de estos, los familiares de los pacientes también son otro punto importante ya que generan mucha presión sobre el personal salud. Se debería monitorizar al personal de salud que presente síntomas de elevado estrés para evitar problemas en su salud mental y en el sistema sanitario que necesita de recursos humanos competentes para combatir la pandemia.

Universidad Nacional Pedro Ruiz Gallo. Lambayeque, Perú.

a Biólogo; Facultad de Ciencias Biológicas, ORCID: https://orcid.org/0000-0002-7418-4856

b Biólogo; Facultad de Ciencias Biológicas, ORCID: https://orcid.org/0000-0002-4984-4656 


\section{Correspondencia:}

Sebastián Iglesias-Osores

Correo electrónico: sebasiglo@gmail.com/siglesias@ unprg.edu.pe

\section{REFERNCIAS BIBLIOGRAFICAS}

1. Park JS, Lee EH, Park NR, Choi YH. Mental health of nurses working at a government-designated hospital during a MERS-CoV outbreak: A crosssectional study. Arch Psychiatr Nurs. 2018;32(1):2-6.

2. Lee AM, Wong JGWS, McAlonan GM, Cheung V, Cheung C, Sham PC, et al. Stress and psychological distress among SARS survivors 1 year after the outbreak. Can J Psychiatry. 2007;52(4):233-40. doi: 10.1177/070674370705200405

3. Lai J, Ma S, Wang Y, Cai Z, Hu J, Wei N, et al. Factors associated with mental health outcomes among health care workers exposed to coronavirus disease 2019. JAMA Netw open. 2020;3(3):e203976. doi: 10.1001/jamanetworkopen.2020.3976

4. Wang W, Tang J, Wei F. Updated understanding of the outbreak of 2019 novel coronavirus (2019-nCoV) in Wuhan, China. J Med Virol. 2020;92(4):441-7. doi: $10.1002 /$ jmv. 25689
5. Kang L, Li Y, Hu S, Chen M, Yang C, Yang BX, et al. The mental health of medical workers in Wuhan, China dealing with the 2019 novel coronavirus. Lancet Psychiatry. 2020;7(3):e14. doi: 10.1016/S2215-0366(20)30047-XThe Lancet Psychiatry. 2020. 7: e14.

6. Maunder R, Hunter J, Vincent L, Bennett J, Peladeau $\mathrm{N}$, Leszcz M, et al. The immediate psychological and occupational impact of the 2003 SARS outbreak in a teaching hospital. CMAJ. 2003;168(10):1245-51.

7. Wu P, Fang Y, Guan Z, Fan B, Kong J, Yao Z, et al. The psychological impact of the SARS epidemic on hospital employees in China: Exposure, risk perception, and altruistic acceptance of risk. Can J Psychiatry. 2009;54(5):302-11.

8. Khalid I, Khalid TJ, Qabajah MR, Barnard AG, Qushmaq IA. Healthcare workers emotions, perceived stressors and coping strategies during a MERS-CoV outbreak. Clin Med Res. 2016;14(1):7-14.

9. DePierro J, Lowe S, Katz C. Lessons learned from 9/11: Mental health perspectives on the COVID-19 pandemic. Psychiatry Res. 2020;288:113024. doi: 10.1016/j.psychres.2020.113024

10. Kang L, Ma S, Chen M, Yang J, Wang Y, Li R, et al. Impact on mental health and perceptions of psychological care among medical and nursing staff in Wuhan during the 2019 novel coronavirus disease outbreak: A cross-sectional study. Brain Behav Immun. 2020.(Por publicarse) doi: 10.1016/j. bbi.2020.03.028 